\title{
Sensitivity Analysis of a Smart 3D-Printed Hand Prosthetic
}

\author{
Ebenezer Olubunmi Ige, ${ }^{1,2}$ Adedotun Adetunla $\mathbb{D}^{1},{ }^{1}$ Adedamola Awesu, ${ }^{1}$ \\ and Oluwaseun K. Ajayi ${ }^{3}$ \\ ${ }^{1}$ Department of Mechanical and Mechatronics Engineering, Afe Babalola University, Ado-Ekiti, Nigeria \\ ${ }^{2}$ Department of Biomedical Engineering, Afe Babalola University, Ado-Ekiti, Nigeria \\ ${ }^{3}$ Department of Mechanical Engineering, Obafemi Awolowo University, Ife, Nigeria
}

Correspondence should be addressed to Adedotun Adetunla; dotunadetunla@yahoo.com

Received 15 November 2021; Revised 4 January 2022; Accepted 2 February 2022; Published 24 February 2022

Academic Editor: Constantin Florin Caruntu

Copyright $(2022$ Ebenezer Olubunmi Ige et al. This is an open access article distributed under the Creative Commons Attribution License, which permits unrestricted use, distribution, and reproduction in any medium, provided the original work is properly cited.

\begin{abstract}
In this study, prosthesis performance was examined in the direction of prosthesis comfort, which may be incorporated into clinical practice as considerations for the fabrication of patient-specific prostheses. The need to produce patient-specific prosthetics is very germane to assist in orthopedic and trans-radial amputation medicine. The prosthesis makes use of a relatively simple braincomputer interface that receives electroencephalogram (EEG) signals as input and drives actuators connected to cables to actuate the 3D-printed fingers and the wrist. Both mechanical and electrical simulations were carried out to investigate the response to loading conditions, after which sensitivity analysis was conducted to validate the prosthesis performance.
\end{abstract}

\section{Introduction}

The human hand is highly functional and inevitable, and in the case of a loss of hand, a replacement should be made possible. Several efforts have been made at providing help to those who have lost a limb or two [1]. The biomechatronics approach is the most recent effort, presenting an engineering approach to solving this medical condition by technologically replicating the limb functionality $[2,3]$. For multiple sclerosis, the use of prosthetic has been found as a suitable solution [4], thus providing a well improved cooperation between the person and the adapted prosthesis would likely decrease stride- stride variation which in turn will improve patient satisfaction. Consequently, the use of hand prosthetics has evolved over the years to a more acceptable solution and is now being gradually domesticated $[5,6]$.

Many amputees abandon their prosthetic arms and wrists because the weight and dimensions of various prostheses can cause discomfort and issues with user coordination; these complications often arise when the prostheses do not fit the amputee properly. In response to this issue, the use of $3 \mathrm{D}$ printers to manufacture prostheses has been introduced $[7,8]$. 3D printing allows for the development of lighter prostheses that can easily be modified to fit the amputee. It is important to take advantage of the new technology provided by $3 \mathrm{D}$ printers to make prostheses affordable for all amputees. Otherwise, amputees who fail to replace their arm or leg may experience complications when they rely on their other arm or leg, such as injuries and arthritis.

However, the use of prosthetics is still very low, especially in underdeveloped countries, due to cost and acceptability. Excitable tissues in the human body generate signals via cells that can be related to electrical potential differences, and these signals are quantifiable, Hence, they are referred to as biopotentials. The characterization of these signals depends on the part of the body where they occur. Among the potentials induced, those from neurons in the brain are called electroencephalogram (EEG); those from muscle cell contraction are called electrocardiogram (ECG); those from skeletal muscle cells are electromyogram (EMG); others are electrooculogram (EOG), neural spikes, and local field potentials (LFPs). Many procedures and devices have 
been developed to detect and capture these signals. EEG equipment has been developed to convert signals from the brain wirelessly through wearable devices to a data acquisition unit unto an actuator to interpret the signal generated and perform required functions [9]. It has helped a great deal and has been extensively used in assisted medical intervention procedures such as implants, diagnosis and diagnostic equipment, and wearable organs and wearable monitoring devices (sensors) [10, 11].

This study is designed to help those who had their hands and/or forearms amputated by developing an affordable, comfortable, yet functional prosthetic hand that can be employed to carry out basic tasks. The prosthesis developed from a $3 \mathrm{D}$ printer makes use of a relatively simple braincomputer interface that receives EEG signals as input and drives actuators connected to cables to actuate the $3 \mathrm{D}$ printed fingers and the wrist. The methodology and explanation are presented in detail.

\section{Methodology}

2.1. Material Selection. The upper limb model from an opensource file was simulated and produced using the biocompatible additive manufactured technique, specifically fusion deposition. The materials used were polylactic acid (PLA) for the phalanges, while ABS was used for the metacarpals and as bolts for the joints. The assembled primitives were enabled for perception and control by signals of electro-encephalogram using an Arduino-based microcontroller to parse the transfer of biopotential signals and a NeuroSky headset used as the brain signal sensor.

2.2. Design Calculations. For easy analysis of the kinematics of the hand under static conditions, a kinematic model composed of 19 links (representing the human bones) and 24 DoFs (represented by joints) was proposed, as shown in Figure 1 [12]. The thumb is modeled as 3 links and 4 joints, while the other fingers are modeled as 4 links and 5 joints. The CMC joint represents the deformation of the palm, e.g., when making a fist.

Four modes of the prosthetic operation were considered, namely: rest, grip, fist, and handshake. Sensitivity analysis carried out showed the sensitivity of the prosthetic forehand to a degree of $20 \mu$ s maximum lag when subjected to armrest and wrist-off loading conditions, which is in line with a similar study [13]. Upon completion of the printing stage, the parts were assembled step by step to form the complete prosthesis. Figure 2 shows the assembly stage in sequential order.

2.3. Control Mechanism. After the assembly was completed, the next step was to place the servomotors in the servo bed designed to be situated in the forearm. A braided fishing line was then run up through each finger individually and looped around one servomotor each. One servomotor was placed in the wrist assembly to aid wrist movement. The lining was then tensioned at two points: when the servos were at $0^{\circ}$ and $90^{\circ}$, as shown in Figure 3. The movement of the phalange was made possible by servo motors positioned to effect each desired movement. The servo motors were connected to the input and output pins on the microcontroller, while variable voltage was achieved through servo motors connected to pulse width modulation (PWM) enabled pins.

2.3.1. Signal Acquisition. NeuroSky offers a wide range of EEG headsets including the MindFlex, MindDuel, and MindWave Mobile headsets. The MindFlex headset was selected for this study due to the relative ease with which it could be integrated with existing devices, as shown in Figure 4.

The MindFlex headset is used to record electrical activity data around the brain. In order for the headset to communicate with the microcontroller, the headset had to be reconfigured. The headset was opened up, the EEG ASIC (called the TGAM) was brought out, and two wires were soldered to the TGAM chip: one to the "T" (transmit) pin of the chip and the other to GND (ground).

2.3.2. Interfacing with the Microcontroller. The six servo motors are connected to digital I/O pins on the Arduino microcontroller and configured as output pins. The wire soldered to the " $\mathrm{T}$ " pin of the EEG headset is connected to another free digital pin on the controller and configured to send data serially to the microcontroller. The GND wire from the headset is connected to a GND pin on the Arduino to reduce the risk of electrical shock. The code for parsing the EEG data from the headset and controlling the BCI was uploaded to the microcontroller, and the electrical circuit configuration is shown in Figure 5.

The flow chart that specifies the sequence of operations of the program is shown in Figure 6, which explains the sequence of operations of the device. The servo has an operating speed of $0.15 \mathrm{sec} / 60^{\circ}$, such that a full wrist rotation from a palm up to a palm down position $\left(180^{\circ}\right)$ was estimated as $0.45 \mathrm{sec}$ using the following equation:

$$
\frac{0.15 s}{60^{\circ}} * 180^{\circ}
$$

It has been measured that a tendon must move about $2 \mathrm{~cm}$ to move the finger from fully extended to be fully flexed, using the arc length

$$
\text { length } \frac{n^{\circ}}{360^{\circ}} * 2 \pi r \text {. }
$$

\section{Results and Discussion}

3.1. Sensitivity Analysis. The arm was controlled by the Arduino and the in-built program has preset algorithms that allow the arm to have different modes. The modes all had specific functions, namely, the rest, grip, fist, and handshake 

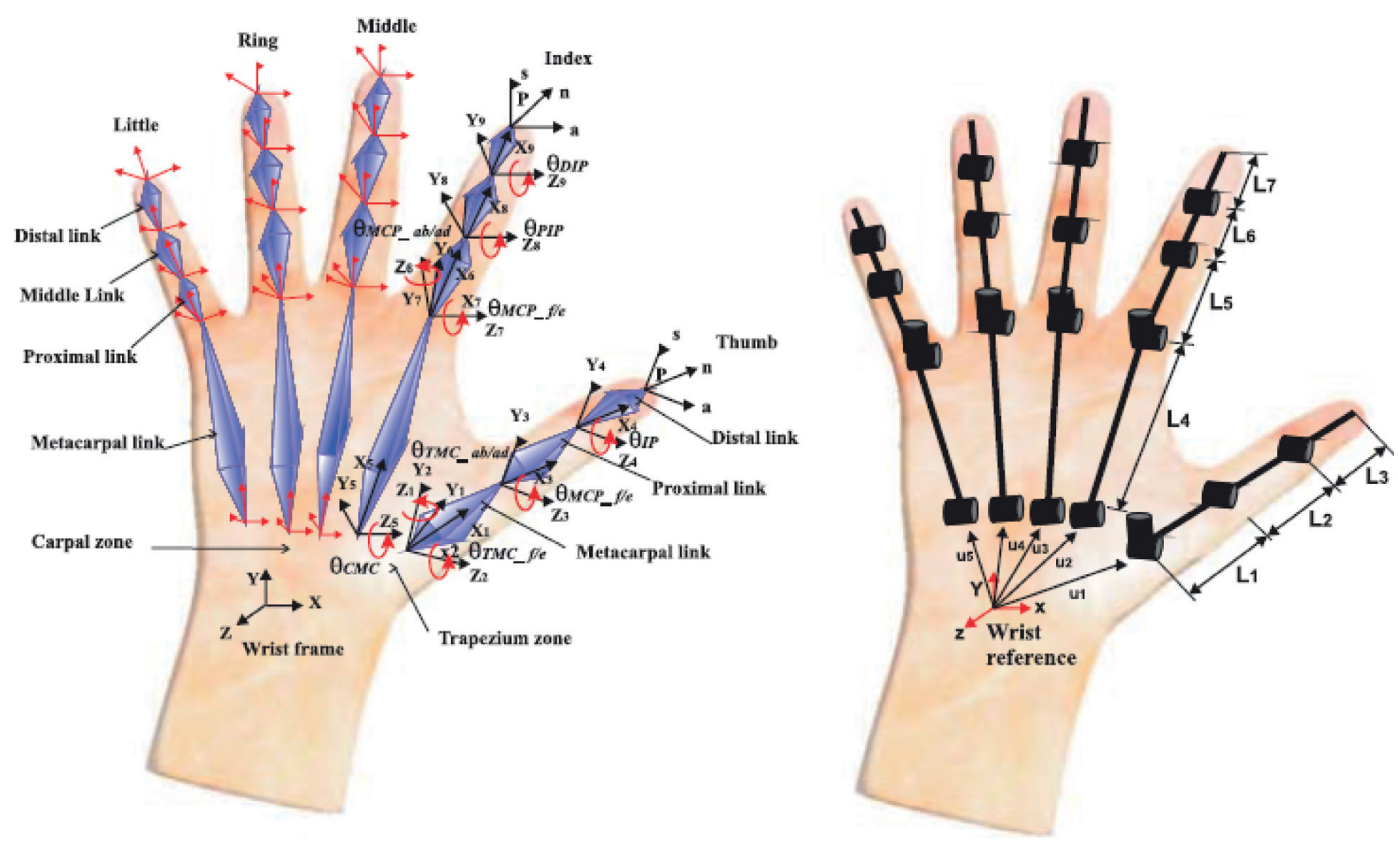

Figure 1: Kinematic configuration of the human hand.

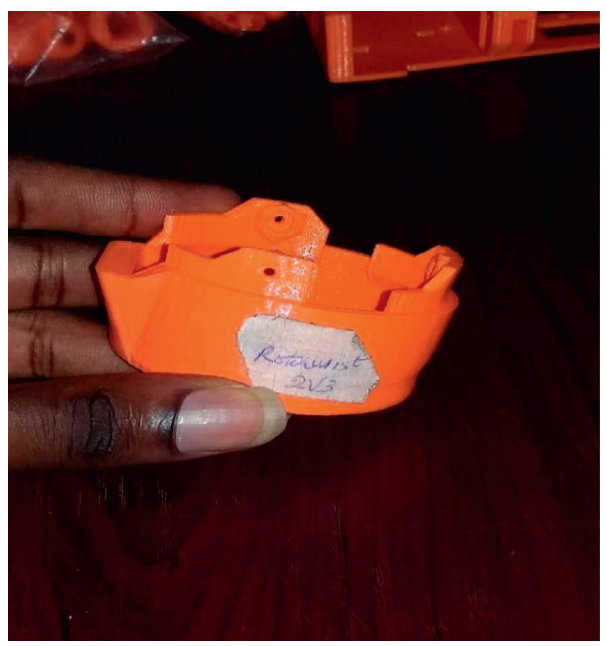

(a)

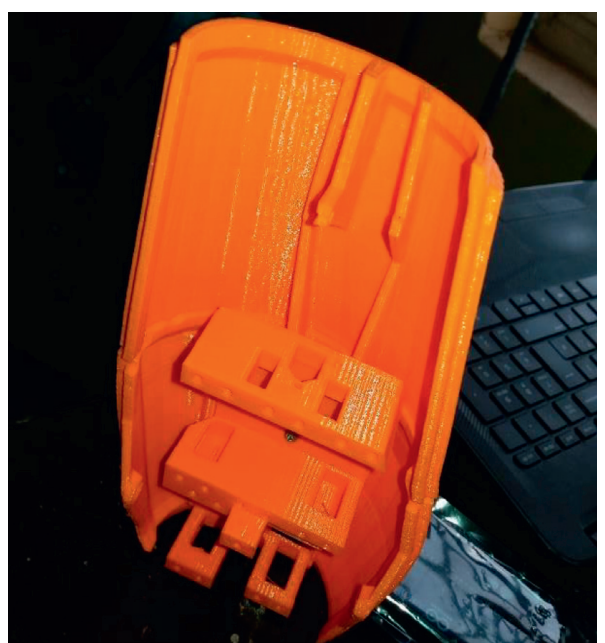

(c)

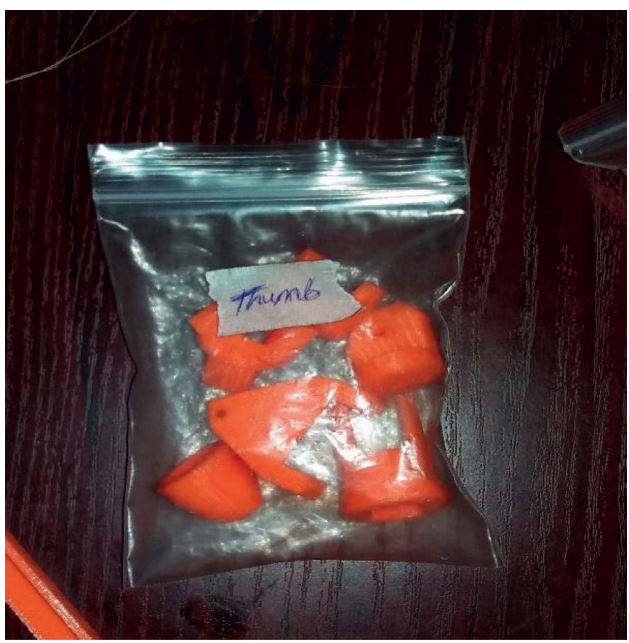

(b)

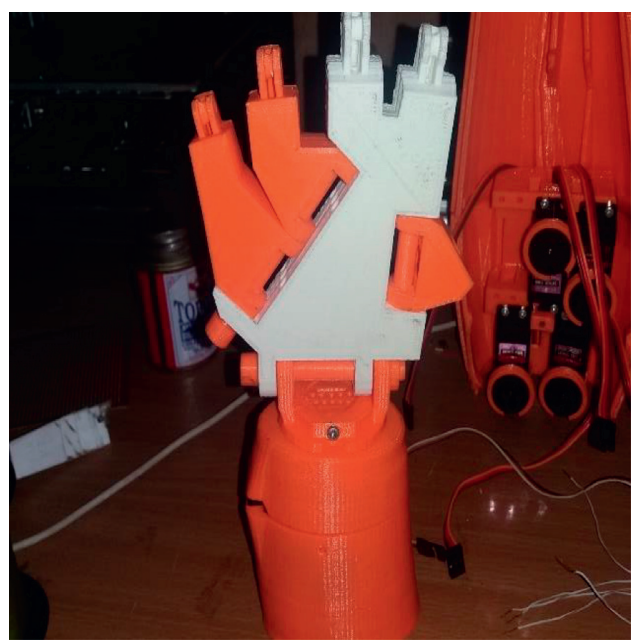

(d)

FIGURE 2: Assembly of the prosthetic hand: (a) wrist component printed, (b) 3D-printed thumb, (c) forearm and servo bed, and (d) wrist and hand assembly. 


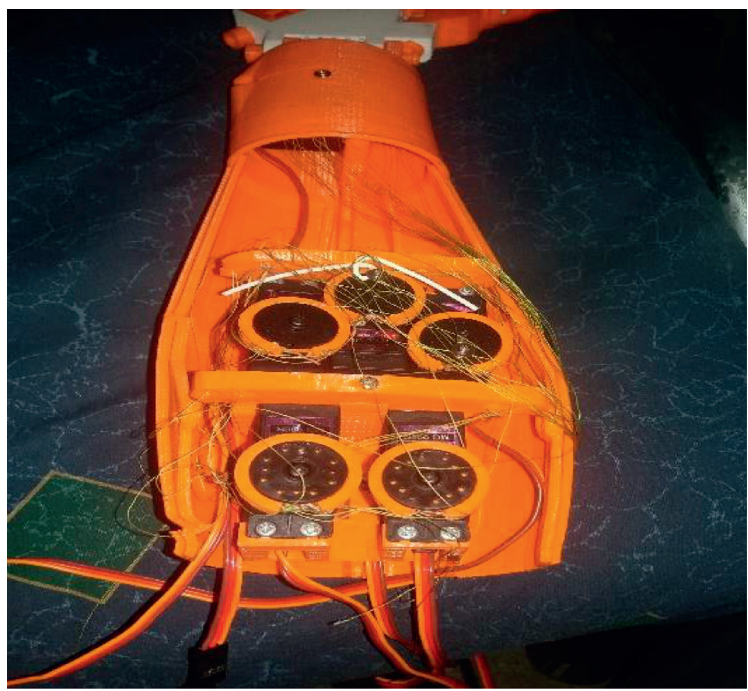

FIGURE 3: Lining and tensioning the hand.

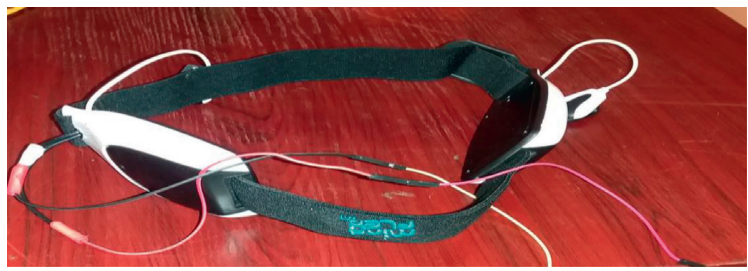

Figure 4: NeuroSky MindFlex headset.

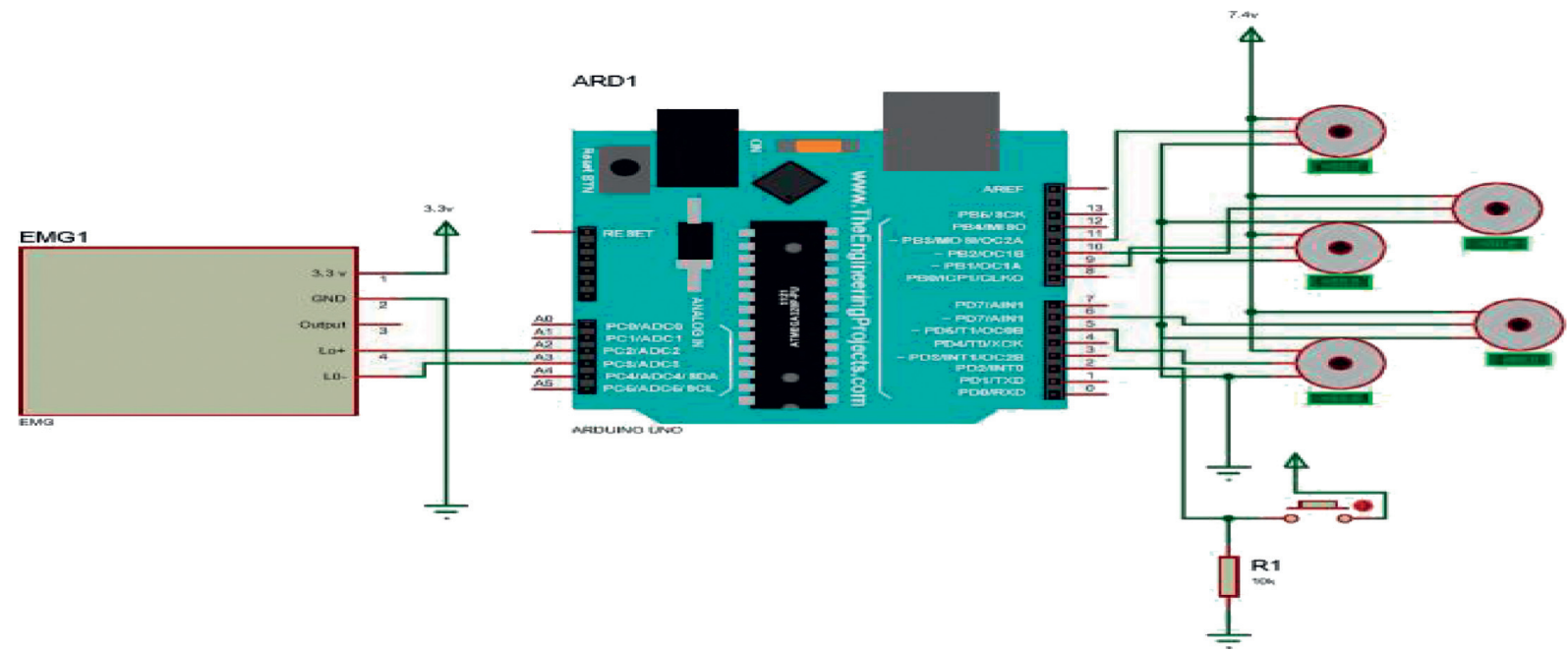

FIgURE 5: Electrical circuit configuration of the prosthesis.

modes. The rest mode is the first mode in which the arm is loaded when it is put on. In this mode, the arm is not performing any functions, the servos are not receiving any signals, and therefore, there is no actuation. The grip mode is used to carry objects. The servos are at $60-70 \%$ actuation, while Figure 7 represents a comparison between the realtime and expected time for a selected loading condition.

A full wrist rotation from a palm up to a palm down position $\left(\left(0.15 \mathrm{~s} / 60^{\circ}\right) * 180^{\circ}\right)$ is estimated as $0.45 \mathrm{sec}$. It has been measured that a tendon must move about $2 \mathrm{~cm}$ to move 


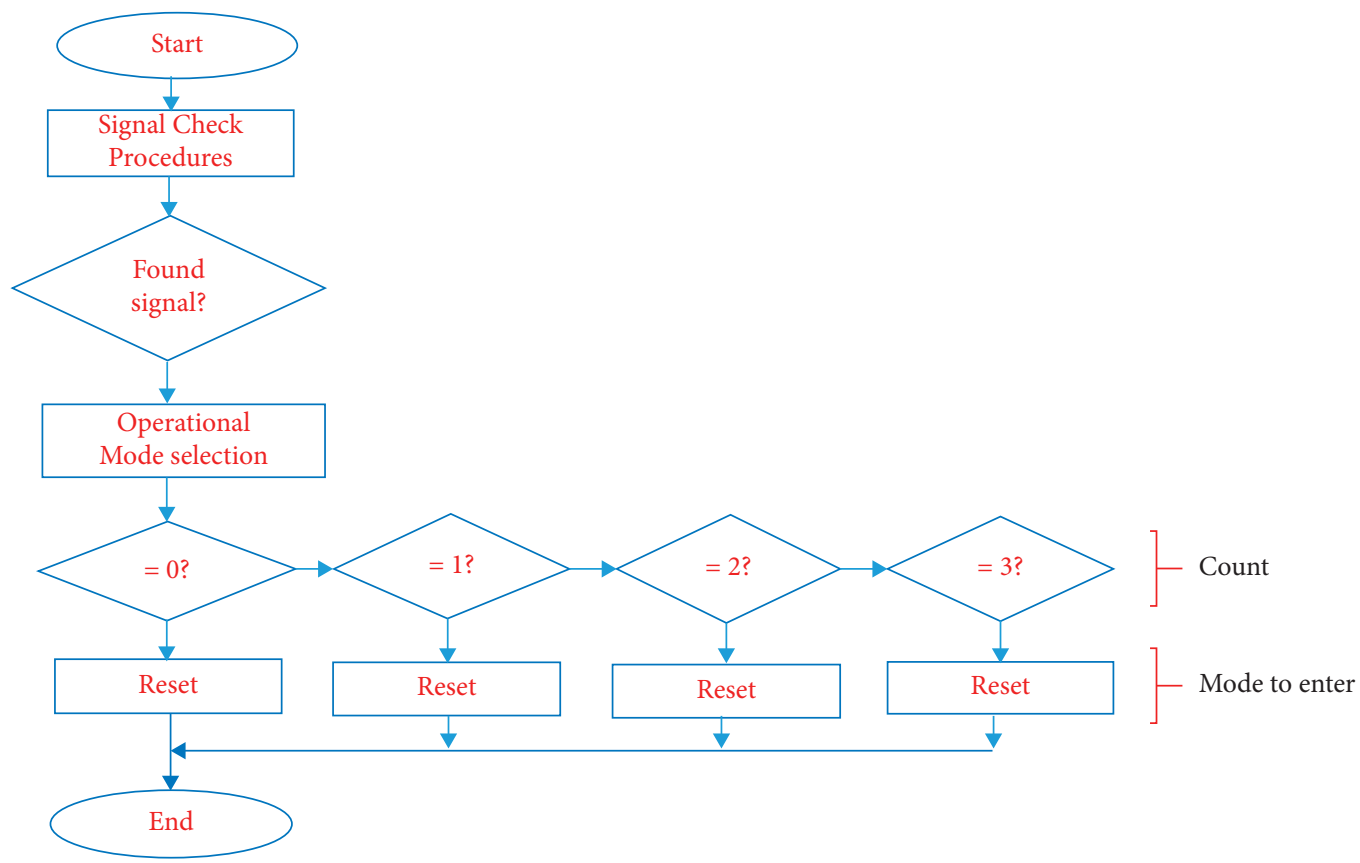

FIGURE 6: Flow chart of the program.

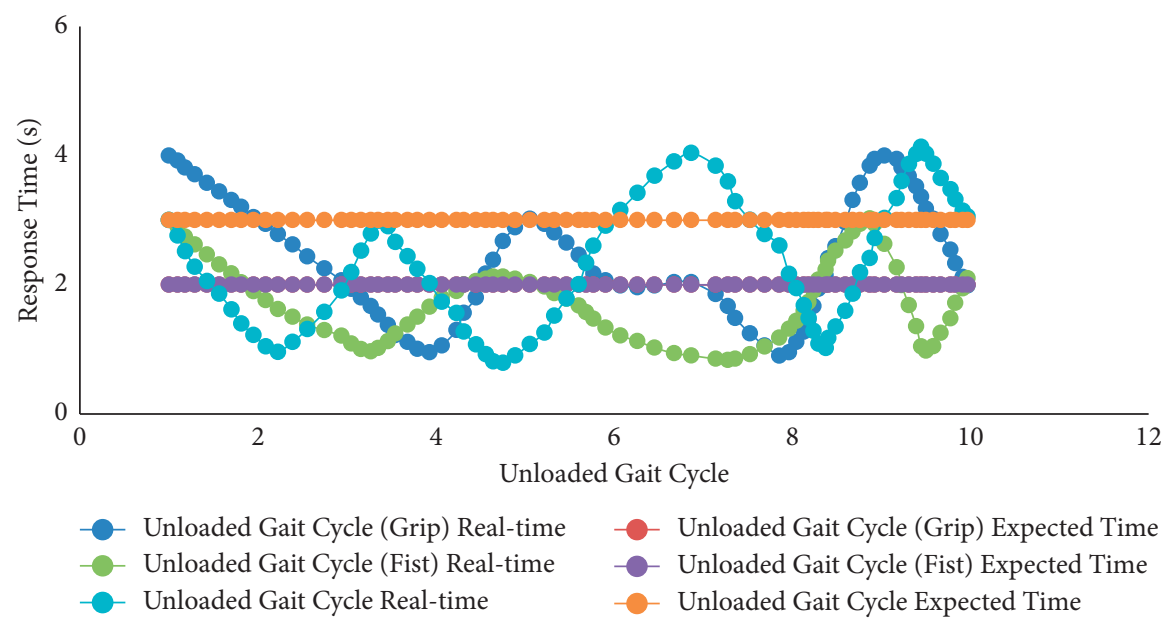

FIGURE 7: Expected time of a grip against real-time response for selected gripping and loading conditions.

the finger from fully extended to be fully flexed, using the arc length $\left(n^{\circ} / 360^{\circ}\right) * 2 \pi r$., where the length is $2 \mathrm{~cm}$ and the radius $r$ of the custom servo horns is $7 \mathrm{~mm}$. It was also found that the servo must rotate by $\left(\left(0.15 / 60^{\circ}\right) * 160^{\circ}\right)$ to completely open/close each finger. We find that the maximum time response to open-close a finger digit is $0.4 \mathrm{sec}$.

3.2. Loading Conditions. For this study, the mechanical parameters under investigation were displacement $(\mathrm{x})$ and Von Mises stresses $(\mathrm{Vm})$ relative to the load applied. Two loading conditions, viz., loading condition 1 (LC1) and loading condition 2 (LC2), were specified for testing: arm rest and wrist off conditions. Simulations were carried out under these two loading conditions, and the results for displacements and stresses followed consistently from the two loading conditions.

3.2.1. Loading Condition 1: "Arm Rest" Conditions. The arm is in rest position. All joints are at a virtual home position. Tables 1 and 2 show the mechanical and electrical parameter combinations for loading condition 1: "Arm rest".

The angular displacement $\theta$ and the response time $t$ of the hand were investigated relative to the voltage output of the motor V. From the graphs of angular displacement and response time against applied voltage in Figure 8, it is seen that for the armrest loading condition, the behavior of the motors is fairly linear. From the results, it is seen that the index, middle, ring, and pinky fingers have very similar 
TABle 1: Mechanical parameter combinations for loading condition 1.

\begin{tabular}{|c|c|c|}
\hline Load applied (F) & $\begin{array}{l}\text { Loading condit } \\
\text { Displacement }(\mathrm{x})\end{array}$ & Von Mises stresses $(\mathrm{Vm})$ \\
\hline \multirow{2}{*}{10} & Max: 8.4306E-6 & $\mathrm{Max}=1.368 \mathrm{E}+5$ \\
\hline & Min: 0 & $\operatorname{Min}=0.8448$ \\
\hline \multirow{2}{*}{20} & Max: $1.6862 \mathrm{E}-5$ & Max: $2.7361 \mathrm{E}+5$ \\
\hline & Min: 0 & Min: 0.8448 \\
\hline \multirow{2}{*}{30} & Max: 2.5292E-5 & $\operatorname{Max}=4.1041 \mathrm{E}+5$ \\
\hline & $\operatorname{Min}=0$ & $\operatorname{Min}=2.5344$ \\
\hline \multirow{2}{*}{40} & Max: $3.3723 \mathrm{E}-5$ & Max: $5.4722 \mathrm{E}+5$ \\
\hline & Min: 0 & Min: 3.3792 \\
\hline \multirow{2}{*}{50} & Max: $4.2154 \mathrm{E}-5$ & Max: $6.8402 \mathrm{E}+5$ \\
\hline & Min: 0 & Min: 4.224 \\
\hline
\end{tabular}

TABLE 2: Electrical parameter combination for loading condition 1.

\begin{tabular}{|c|c|c|c|c|c|c|c|c|c|c|c|}
\hline & \multirow{2}{*}{ Voltage applied (V) } & \multicolumn{5}{|c|}{ Angular displacement $(\theta)$} & \multicolumn{5}{|c|}{ Response time $(\mathrm{t})$} \\
\hline & & Thumb & Index & Middle & Ring & Pinky & Thumb & Index & Middle & Ring & Pinky \\
\hline \multirow{5}{*}{ Loading condition 2} & 2.22 & 36.27 & 35.62 & 35.65 & 35.64 & 35.65 & 0.70 & 0.69 & 0.70 & 0.68 & 0.68 \\
\hline & 4.44 & 71.69 & 72.25 & 72.22 & 72.23 & 72.25 & 0.75 & 0.77 & 0.76 & 0.76 & 0.75 \\
\hline & 6.66 & 108.02 & 108.09 & 108.07 & 108.10 & 108.06 & 0.87 & 0.86 & 0.87 & 0.87 & 0.85 \\
\hline & 8.88 & 143.67 & 143.75 & 143.77 & 143.78 & 143.75 & 0.92 & 0.91 & 0.94 & 0.93 & 0.93 \\
\hline & 11.1 & 179.90 & 179.90 & 179.86 & 179.91 & 179.90 & 1.02 & 1.01 & 1.03 & 0.99 & 0.98 \\
\hline
\end{tabular}

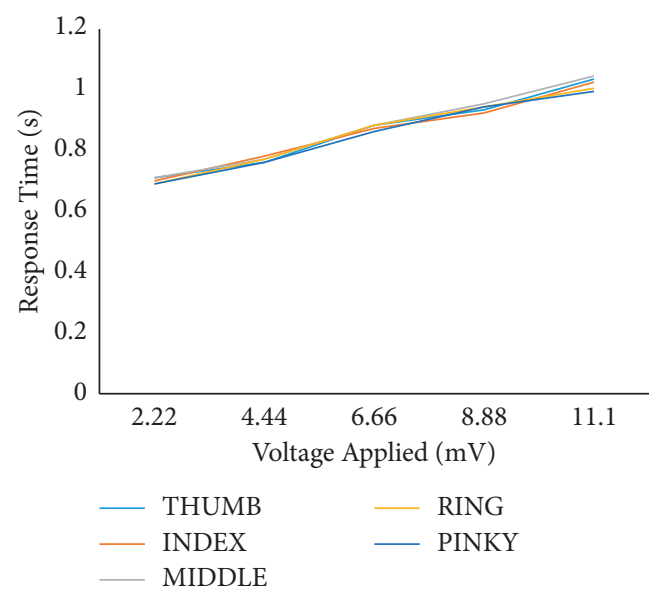

(a)

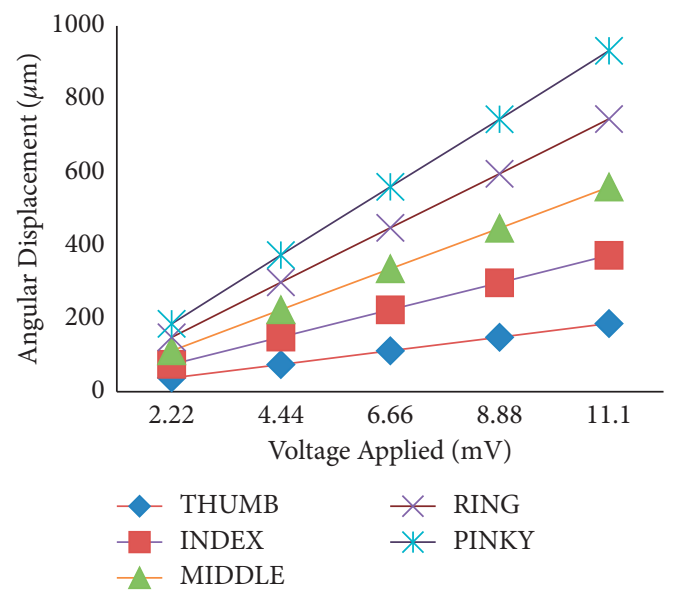

(b)

FIgURe 8: (a) Plot of angular displacement against voltage for LC1 and (b) plot of response time against voltage for loading condition 1.

responses to mechanical and electrical excitation. This justifies modeling the middle, ring, and pinky fingers using the index.

3.2.2. Loading Condition 2: "Wrist Off" Conditions. The wrist is at $120^{\circ}$ from the $x$-axis (elevated from the rest position). Parameter combinations are shown in Tables 3 and 4 for the loading conditions.

For the wrist-off loading condition, the angular displacement displays the same fairly linear characteristics as with the arm rest condition (LC1); the response time, however, is seen to deviate from that pattern and take on a slightly triangular function, as shown in Figure 9. It can be inferred from these results that the index, middle, ring, and pinky fingers have very similar responses to mechanical and electrical excitation. Simulation and experimental analysis revealed that the magnitude of bearing loads of 10 and $50 \mathrm{~N}$ generated maximum stresses in the range of $6.8402 \mathrm{E}+5$ and $6.0261 \mathrm{E}+5$, respectively. The angular displacement of the five digits for an applied voltage ranging from 2 to $10 \mathrm{~V}$ was obtained as $36.27^{\circ}$ to $179.90^{\circ}$ and $34.55^{\circ}$ to $175.93^{\circ}$, respectively.

Figure 10 shows the Von Mises stresses and directional displacement of the hand caused by different loads under the specified loading conditions. 
TABLE 3: Mechanical parameter combination for loading condition 2.

\begin{tabular}{|c|c|c|}
\hline \multicolumn{3}{|c|}{ Loading condition 2} \\
\hline Load applied (F) & Displacement (x) & Von Mises stresses $(\mathrm{Vm})$ \\
\hline \multirow{2}{*}{10} & Max: 7.67754E-6 & $\mathrm{Max}=1.2052 \mathrm{E}+5$ \\
\hline & Min: 0 & $\operatorname{Min}=1.0184$ \\
\hline \multirow{2}{*}{20} & Max: $1.5351 E-5$ & Max: $2.4104 \mathrm{E}+5$ \\
\hline & Min: 0 & Min: 2.0368 \\
\hline \multirow{2}{*}{30} & Max: $2.3026 \mathrm{E}-5$ & $\operatorname{Max}=3.6156 \mathrm{E}+5$ \\
\hline & $\operatorname{Min}=0$ & $\operatorname{Min}=3.0551$ \\
\hline \multirow{2}{*}{40} & Max: $3.0702 \mathrm{E}-5$ & Max: $4.8209 \mathrm{E}+5$ \\
\hline & Min: 0 & Min: 4.0735 \\
\hline \multirow{2}{*}{50} & Max: 3.8377E-5 & Max: $6.0261 \mathrm{E}+5$ \\
\hline & Min: 0 & Min: 5.0919 \\
\hline
\end{tabular}

TABLE 4: Electrical parameter combination for loading condition 2.

\begin{tabular}{|c|c|c|c|c|c|c|c|c|c|c|c|}
\hline & \multirow{2}{*}{ Voltage applied (V) } & \multicolumn{5}{|c|}{ Angular displacement $(\theta)$} & \multicolumn{5}{|c|}{ Response time $(\mathrm{t})$} \\
\hline & & Thumb & Index & Middle & Ring & Pinky & Thumb & Index & Middle & Ring & Pinky \\
\hline \multirow{5}{*}{ Loading condition 2} & 2.22 & 36.15 & 34.22 & 34.55 & 34.37 & 34.35 & 0.96 & 0.92 & 0.93 & 0.92 & 0.91 \\
\hline & 4.44 & 70.53 & 70.86 & 70.89 & 70.91 & 70.82 & 1.09 & 1.14 & 1.15 & 1.16 & 1.14 \\
\hline & 6.66 & 108.02 & 107.51 & 107.53 & 107.57 & 107.56 & 1.47 & 1.44 & 1.44 & 1.45 & 1.45 \\
\hline & 8.88 & 141.67 & 141.63 & 141.60 & 141.59 & 141.59 & 1.66 & 1.66 & 1.66 & 1.65 & 1.67 \\
\hline & 11.1 & 175.92 & 175.90 & 175.92 & 175.89 & 175.93 & 1.72 & 1.71 & 1.73 & 1.71 & 1.73 \\
\hline
\end{tabular}

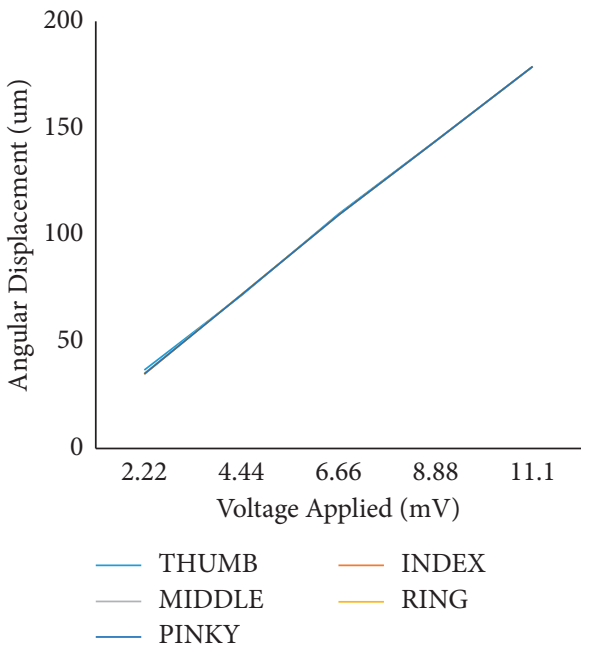

(a)

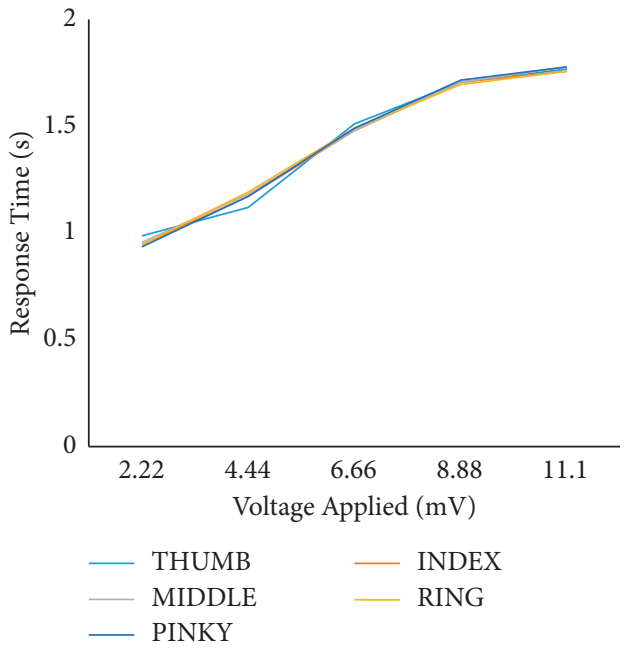

(b)

FIgURe 9: (a) Plot of displacement against voltage for loading condition 2 and (b) plot of response time against voltage for loading condition 2 .

3.3. Prosthetic Performance and Acceptability. The bioprosthetic was rated based on its evaluative behavior when made to perform some grips, fists, and shake functions. Ten users were selected, and each one was made to use the bioprosthesis in the three modes of operation as well. Using a baseline of 2 seconds as the expected time for the prosthesis to perform any of the three functions as presented in Figure 11, it was observed that the prosthesis performed fairly around this value. The real-time average is $2.4 \mathrm{sec}$ with an expected time of $2.0 \mathrm{sec}$. The deviation falls within an acceptable range of $0.4 \mathrm{sec}$.

The prosthesis presented in this paper makes use of a relatively simple brain-computer interface that receives EEG signals as input and drives actuators connected to cables to actuate the $3 \mathrm{D}$-printed fingers, and the wrist is presented in Figure 12; this helps for the integration of the whole system. 


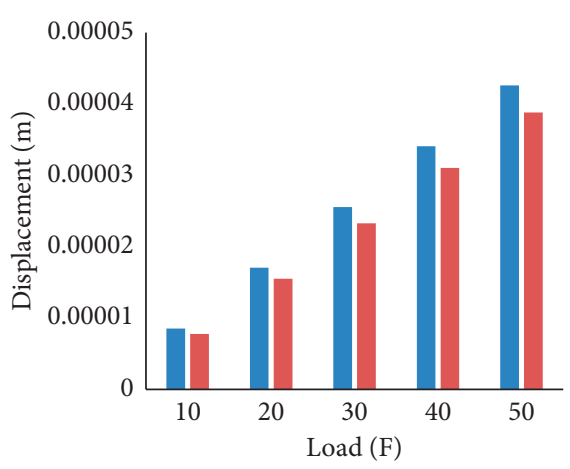

DISPLACEMENT (LC1)

DISPLACEMENT (LC2)

(a)

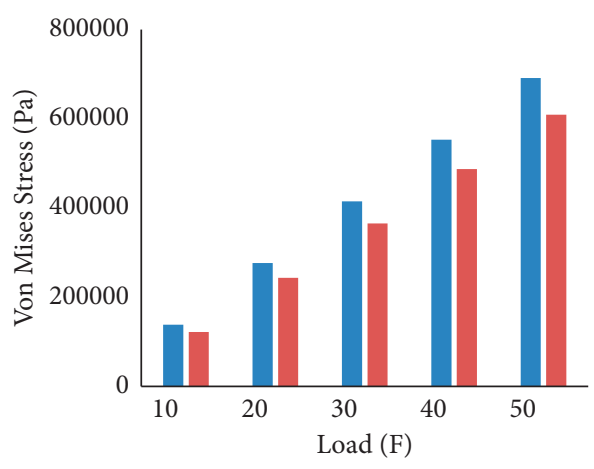

Von Mises Stress (LC1)

- Von Mises Stress (LC2)

(b)

Figure 10: (a) Plot of displacement against load for LC1 and LC2 and (b) plot of Von Mises stress against load for LC1 and LC2.

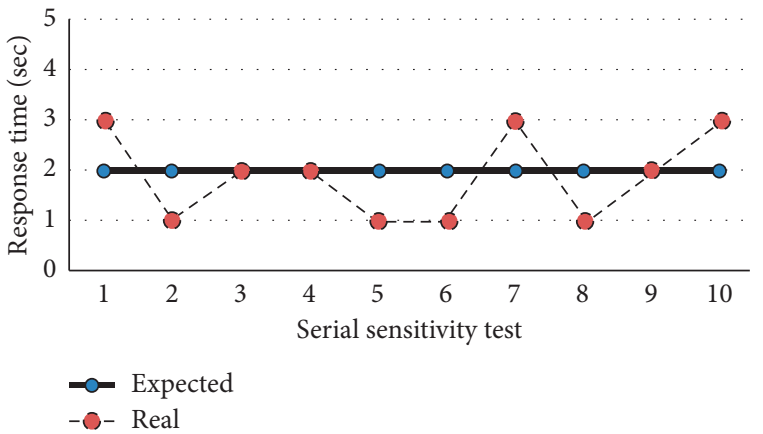

(a)

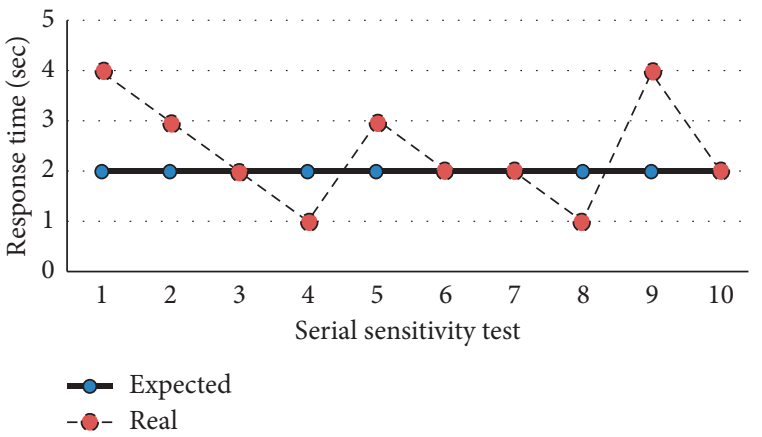

(b)

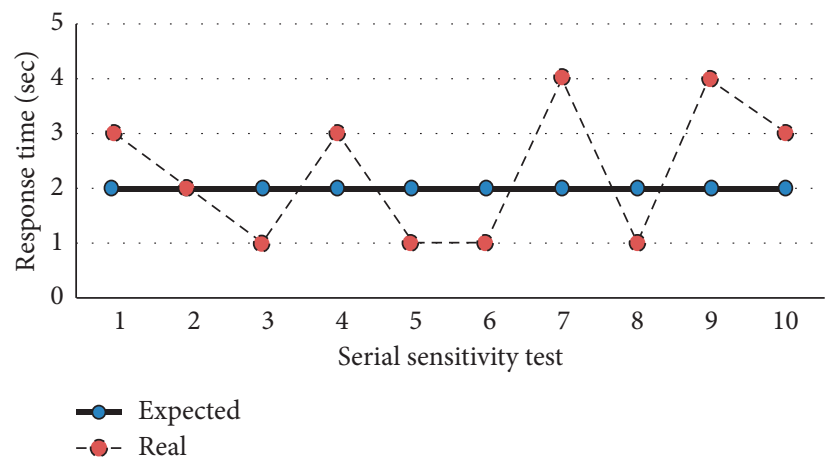

(c)

FIGURE 11: (a) Expected time of sensing for the fist mode, (b) expected time of sensing for the grip mode, and (c) expected time of sensing for the handshake mode.

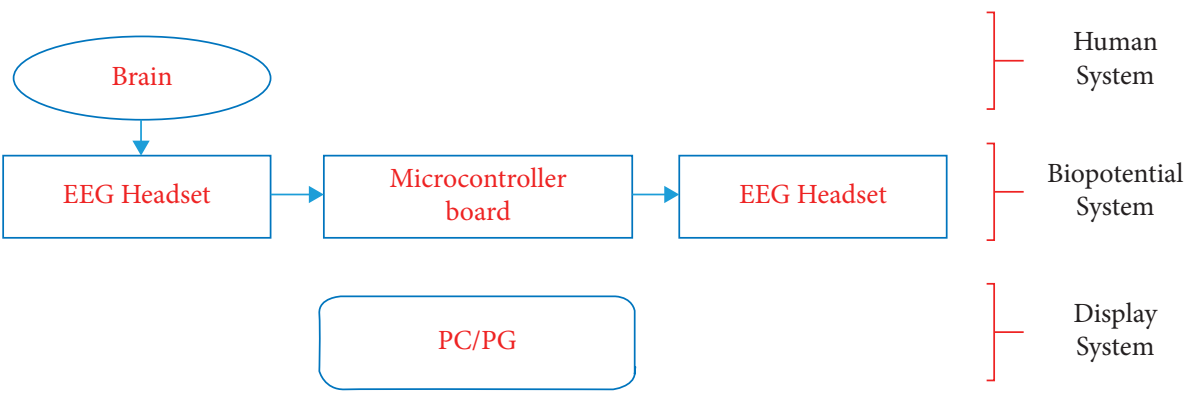

FIgURE 12: Schematic of the EEG-controlled prosthesis. 


\section{Conclusions}

This study demonstrates the performance of prosthesis fabricated via $3 \mathrm{D}$ printing technology with the aim to build a prosthetic limb and control it with EEG biopotential. This study presents the development of a biopotential-controlled transradial prosthesis for people suffering from transradial upper-limb extremities either as a result of injury or congenial conditions. The limb was successfully fabricated using a 3D printer, and the EEG signal was recorded from the scalp and used to control the hand to open or close.

The capability of 3D bio-plastic design was demonstrated to show the prosthetic performance and the sensitivity of trans-radial polymer-grade prosthesis with capabilities of harvesting biopotential signals and effecting the actuation of digits of the upper extremities (upper arm, forearm, and hand) to assume load-carrying capacity. The developed prosthesis can be adapted for various users and is recommended for use in hospitals where corrective and cosmetic surgeries are performed.

\section{Data Availability}

The data used to support this study are included within the article.

\section{Conflicts of Interest}

The authors declare that they have no conflicts of interest.

\section{References}

[1] C. Cipriani, M. Controzzi, and M. C. Carrozza, "The SmartHand transradial prosthesis," Journal of NeuroEngineering and Rehabilitation, vol. 8, no. 1, p. 29, 2011.

[2] A. M. Dollar and R. D. Howe, "The highly adaptive SDM hand: design and performance evaluation," The International Journal of Robotics Research, vol. 29, no. 5, pp. 585-597, 2010.

[3] S. Amaral and L. S. Levin, "Pediatric and congenital hand transplantation," Current Opinion in Organ Transplantation, vol. 22, no. 5, pp. 477-483, 2017.

[4] T. Zhang, X. Q. Wang, L. Jiang et al., "Biomechatronic design and control of an anthropomorphic artificial hand for prosthetic applications," Robotica, vol. 34, no. 10, pp. 2291-2308, 2016.

[5] A. D. Deshpande, Z. Xu, M. J. V. Weghe et al., "Mechanisms of the anatomically correct testbed hand," IEEE, vol. 18, no. 1, pp. 238-250, 2013.

[6] D. Xing, C.-I. Yeh, and R. M. Shapley, "Spatial spread of the local field potential and its laminar variation in visual cortex," Journal of Neuroscience, vol. 29, no. 37, pp. 11540-11549, 2009.

[7] C. Dally, D. Johnson, M. Canon, S. Ritter, and K. Mehta, "Characteristics of a 3D-printed prosthetic hand for use in developing countries," in Proceedings of the IEEE Global Humanitarian Technology Conference (GHTC), pp. 66-70, USA, October 2015.

[8] J. Koprnicky, P. Najman, and J. Safka, "3D printed bionic prosthetic hands," in Proceedings of the 2017 IEEE International Workshop of Electronics, Control, Measurement, Signals and their Application to Mechatronics (ECMSM), Donostia, Spain, May 2017.
[9] C. Liu, F. Liu, L. Zhang, Y. Su, and A. Murray, "Smart wearables in healthcare: signal processing, device development, and clinical applications," Journal of Healthcare Engineering, vol. 2018, Article ID 1696924, 2 pages, 2017.

[10] D. R. Seshadri, R. T. Li, J. E. Voos et al., "Wearable sensors for monitoring the physiological and biochemical profile of the athlete," NPJ Digital Medicine, vol. 2, no. 1, p. 72, 2019.

[11] A. Adetunla and E. Akinlabi, "Influence of reinforcements in friction stir processed magnesium alloys: insight in medical applications," Materials Research Express, vol. 6, no. 2, 2018.

[12] S. Cobos, M. Ferre, M. A. Sanchéz-Urán, J. Ortego, and C. Peña, "Efficient human hand kinematics for manipulation tasks," in Proceedings of the 2008 IEEE/RSJ Intelligent Robots and Systems, pp. 2246-2251, France, October 2008.

[13] N. Baneshi, B. K. Moghadas, A. Adetunla et al., "Investigation the mechanical properties of a novel multicomponent scaffold coated with a new bio-nanocomposite for bone tissue engineering: fabrication, simulation and characterization," Journal of Materials Research and Technology, vol. 15, pp. 5526-5539, 2021. 